\title{
A Deep CNN Model for Skin Cancer Detection and Classification
}

\author{
Masum Shah Junayed \\ Bahcesehir University \\ Istanbul 34439, Turkey \\ masumshahjunayed@gmail.com
}

\author{
Nipa Anjum, Abu Noman \\ Md Sakib \\ Khulna University of \\ Engineering \& Tech. \\ Khulna, Bangladesh
}

\author{
Md Baharul Islam \\ American University of Malta \\ Bormla 1013, Malta \\ bislam.eng@gmail.com
}

\begin{abstract}
Skin cancer is one of the most dangerous types of cancers that affect millions of people every year. The detection of skin cancer in the early stages is an expensive and challenging process. In recent studies, machine learning-based methods help dermatologists in classifying medical images. This paper proposes a deep learning-based model to detect and classify skin cancer using the concept of deep Convolution Neural Network (CNN). Initially, we collected a dataset that includes four skin cancer image data before applying them in augmentation techniques to increase the accumulated dataset size. Then, we designed a deep CNN model to train our dataset. On the test data, our model receives $95.98 \%$ accuracy that exceeds the two pre-train models, GoogleNet by $1.76 \%$ and MobileNet by $1.12 \%$, respectively. The proposed deep CNN model also beats other contemporaneous models while being computationally comparable.
\end{abstract}

\section{Keywords}

Skin Cancer, Dataset, Data Augmentation, Deep CNN, Medical Image, Computer Vision.

\section{INTRODUCTION}

An uncontrollable growth of abnormal cells that appears on our skin is called skin cancer. It happens when some unusual DNA damage activates mutations [1] that helps skin cells to increase very fast, and this forms malignant tumours. Some major skin cancer types are basal cell carcinoma, squamous cell carcinoma, actinic keratosis and malignant melanoma. Basal cell carcinoma is a nonmelanoma type cancer that starts with different sized nodules [1]. The second type of skin cancer is squamous cell carcinoma that creates scaly red marks, open inflammations, uneven clots or lumps in the skin. In the U.S., more than 1 million cases of this type are diagnosed each year [2]. A different kind of skin cancer is actinic keratosis that is the initial stage of squamous cell carcinoma [3]. The last one is a serious one among others which is malignant melanoma. It occurs when pigment cells grow without any control. Without any indication, 90\% of melanomas are diagnosed as primary tumours [4].

Permission to make digital or hard copies of all or part of this work for personal or classroom use is granted without fee provided that copies are not made or distributed for profit or commercial advantage and that copies bear this notice and the full citation on the first page. To copy otherwise, or republish, to post on servers or to redistribute to lists, requires prior specific permission and/or a fee.
Ultraviolet radiation triggers these tumours to increase. These cancer cells are seen mostly on sun-exposed areas like ear, lips, face, around the eye, scalp, neck, hand. American Cancer Society [5] estimated the death rate for melanoma skin cancer to be 6850 , where new cases of this estimation are 100,350 where 60,190 people are male, and 40160 are female. They also estimated that the death rate is higher in male than female. A specialist do visual analysis by analysing the pigmented lesions by changing size, irregular shape and colour. Then histopathologic diagnosis is another way to examine the cancer cell. Here experienced dermatopathologist help to determine the melanoma. The earlier cancer detection can be useful to save many lives. Vocaturo et al. [6] have shown different machine learning techniques for automatically detecting melanoma. These techniques help to research more on skin cancers [7]. However, we need systems that can accurately predict skin cancer or not and its type.

Non-pigmented lesions like basal cell carcinoma, squamous cell carcinoma, actinic keratosis and pigmented lesion like malignant melanoma have some clinical differences: bleeding, pain, and itching. But the appearance of these lesions on human body parts is very similar. So it is difficult to distinguish any of these skin cancers. Besides visual analysis, an automated system can help a physician find skin cancer types faster and easier and reduce patient life risk. Classification algorithms such as K-nearest neighbour, decision tree, deep learning, [8] logistic regression, support vector machine etc. 
are used to describe how well these classifiers perform on dermoscopic images. In [9], K-mean clustering is used to segment skin cancer, and then features are extracted using grey level co-occurrence matrix. The support vector machine is used as a classifier. For faster and better prediction, A classification technique can also help the patient life that relies on the detection result [11] [12] [13] [10]. It motivates us to implement a deep learning approach to build a CNN model that can classify four different skin cancer types.

This paper proposes a deep convolutional neural network model that automatically detects and classifies actinic keratosis, basal cell carcinoma, malignant melanoma, and squamous cell carcinoma. To test our model, we collect a dataset and train it in our model with other two pre-train models. Our model provides satisfying accuracy compare to the state of the arts. The summary of our contribution is four folded.

- We collect a skin cancer dataset, size 800 images of four skin cancer classes that are, Actinic Keratosis, Basal Cell Carcinoma, Malignant Melanoma and Squamous Cell Carcinoma. We use data augmentation techniques for increasing the dataset at 5600 images that were split into training and test set for deep CNN models.

- A deep CNN model has been proposed that has convolution layers for extracting features and fully connected layers for classifying the cancer type. Regularization techniques like batch-normalization and dropout helped to reduce the overfitting.

- We present a comparative study of two pre-trained models that shows how our model differs from architecture and accuracy.

Rest of the paper is discussed as follows: Section 2 most related works, Section 3 our proposed deep CNN model, Section 4 experimental results and discussion, and we conclude our work with further research direction in Section 5 .

\section{RELATED WORKS}

This section narrates some most related works on Skin Cancer using different techniques for detecting and classifying.

\subsection{Learning based Approaches}

Garg et al. [14] proposed a way to detect melanoma skin cancer by using some image processing techniques. They have implemented an ABCD rule called Asymmetry, Border Irregularity, Color, and Diameter. An illumination correction used before the skin lesion segmentation. They received the accuracy up to
91.6\%. Tammineni et al. [15] proposed a melanoma segmentation technique for early detection of skin cancer. A melanoma segmentation technique used based on Gradient and Feature Adaptive Contour (GFAC) model. They experimented on PH2 dataset consisting of 200 images. The achieved accuracy for the proposed segmentation techniques is $98.64 \%$. The acquired accuracy is well enough, but it is limited to a low sized dataset.

\subsection{CNN based Approaches}

Pham et al. [16] classified skin lesions with Deep CNN with Data Augmentation. They combined images from different sources such as ISBI Challenge, ISIC Archive, $\mathrm{PH} 2$ dataset to prepare the dataset. InceptionV4 was used as the model architecture, and the outcomes compared by using Support Vector Machine (SVM), Random Forest (RF) and Neural Network (NN) as classifiers. They achieved an overall accuracy of $89 \%$. Even though the model architecture was InceptionV4, the gained accuracy was not satisfactory. Jordan et al. [17] proposed a method to classify multimodal skin lesion using deep learning. They have composed their dataset with 2917 cases from five classes. They have used modified ResNet-50 as the model architecture. As a modification, they removed the softmax and fully connected layer from the end. They used 2048-dimensional image feature vector as the flattened output, which they referred to as an image feature extraction network. They received $85.8 \%$ average accuracy in single image classification, and the highest multimodal network accuracy was $86.6 \%$. The multimodal network fails to acquire higher accuracy. Serban et al. [18] implemented a convolutional neural network to facilitate automatic diagnosis of skin cancer. The dataset consists of 1000 images collected from International Skin Imaging Collaboration and $\mathrm{PH} 2$ databases. There are two classes, benign tumours and skin malignant lesions, each having 500 images. Then the dataset was fed to the neural network. The proposed solution acquired $80.52 \%$ accuracy on the dataset.The dataset has only two classes and the accuracy can be improved.

Hosny et. al [19] classified three different types of skin cancer that are melanoma, common nevus and atypical nevus. They modified the AlexNet architecture. When the model was trained with original images, they got $80 \%$ accuracy. The accuracy was $98.61 \%$ after using augmented images. They used 200 images from ph2 dataset which became 11000 after applying augmentation. However, there are 11000 images after augmentation while the original dataset has three classes with 200 images in total. Ensaf et al. [20] have used transfer learning as a part of deep convolutional networks to classify enhanced skin lesions. They used HAM10000 dataset. In the pre-processing step, the 
dataset was cleaned, downsampled, split and then augmented. As model architecture, DenseNet-121 and MobileNet architecture was used. Both CNN models were pre-trained. After evaluation, DenseNet-121 and MobileNet achieved $71.9 \%$ and $82.6 \%$ testing accuracy, respectively on the unbalanced dataset. Whereas, they reached $92.7 \%$ and $91.2 \%$ accuracy, respectively on the balanced dataset. It was an impressive improvement.

Nahata et. al [21] proposed skin cancer detection model using deep learning that can classify the types of skin cancer. They used similar seven classes from ISIC 2018 and 2019 dataset. Transfer learning was used to train the CNN. For this, InceptionV3, ResNet50, VGG16, MobileNet and InceptionResnet was used. Among all these models, InceptionResnet achieved $91 \%$ accuracy. The dataset had 35,348 images with seven classes. Improvement in the model architecture can give more accurate prediction result. Ulzii et al. [22] proposed a way to classify skin cancer by using ECOC SVM classifier with pre-trained AlexNet architecture. They collected the dataset through searching on different search engines available online. The dataset consists of 3753 images with 4 classes Actinic Keratoses (897), Melanoma (958), Squamous cell carcinoma (977), Basal cell carcinoma (921). For the model architecture, pre-trained AlexNet model was used to extract the training features. The classifier was Error-Correcting Output Codes (ECOC) SVM. After evaluation, they were able to acquire $94.2 \%$ accuracy on multiclass classification. Further improvement can increase the accuracy of their model.

The earlier research was mostly focused on melanoma, but research focusing on various skin cancer types is scarce. Our focus is on improving the dataset and building a model that can classify different types of skin cancer.

\section{METHODOLOGY}

In this section, we have described the overall workflow of our proposed method. At first, we have collected a dataset then we preprocessed the dataset with the augmentation method. Finally, we proposed a deep learning-based model to detect skin cancer, as shown in Figure 2

\subsection{Dataset}

Our data collection process was a challenging task. We collect 21 images from the Department of Dermatology, Dhaka Medical College (DMC), Dhaka, Bangladesh. Other 779 images acquired from www.dermnet.com, which is the largest independent photo dermatology source dedicated to online medical education. Our dataset contains four different classes of Skin Cancer, including Actinic
Keratosis (AK), Basal Cell Carcinoma (BCC), Malignant Melanoma (MM) and Squamous Cell Carcinoma (SCC). Figure 1 shows a sample of our dataset on skin cancer. After collecting the dataset, we resized our dataset because all the images were not the same size. So, the images were pre-processed by resizing them into $224 \times 224$ pixel as it gives a better result. For our deep learning approach, the number of images was not enough for the model's learning.
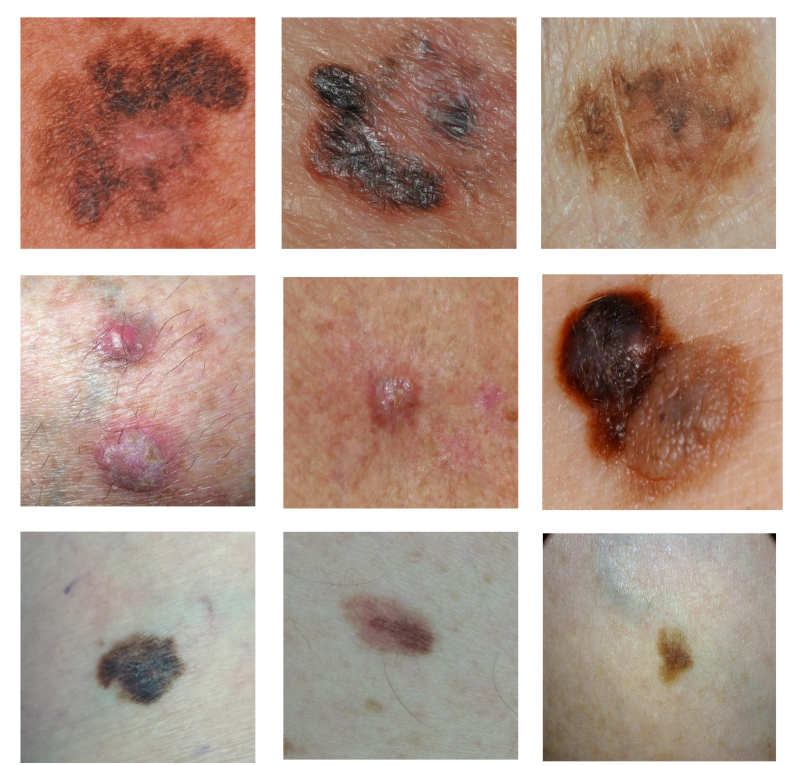

Figure 1: A sample of our collected dataset.

Table 1: Image Augmentation Parameters.

\begin{tabular}{|l|l|}
\hline $\begin{array}{l}\text { Transformation } \\
\text { Types }\end{array}$ & Descriptions \\
\hline Rotation & $\begin{array}{l}\text { Rotate an angle ranging from 0 to 360 de- } \\
\text { grees at random. }\end{array}$ \\
\hline Flipping & (Without) or 1 (With) flipping \\
\hline Re-scaling & $\begin{array}{l}\text { With a scale factor ranging from 0.3 to } \\
0.8, \text { at random }\end{array}$ \\
\hline Shading & $\begin{array}{l}\text { From the middle to the edges, the image } \\
\text { brightness is reduced. }\end{array}$ \\
\hline Translation & $\begin{array}{l}\text { Adding a given value to the x-axis and y- } \\
\text { axis shifts the image in coordinate space. }\end{array}$ \\
\hline Shearing & $\begin{array}{l}\text { Shifting one portion of the image in the } \\
\text { manner of a parallelogram. }\end{array}$ \\
\hline
\end{tabular}

\subsection{Pre-processing and Augmentation}

We used different augmentation techniques to improve the dataset size using rotation, flipping, shading, translation, shearing and scaling. We applied 0.2 as shifting, shear and zoom range. Rotation transforms a source image by rotating clockwise or counterclockwise by some number of degrees randomly. Flipping means the rotation of an image in horizontal or vertical axis. Shading is done by different hue values denoting the shade of colors in the image. Translation means moving the 


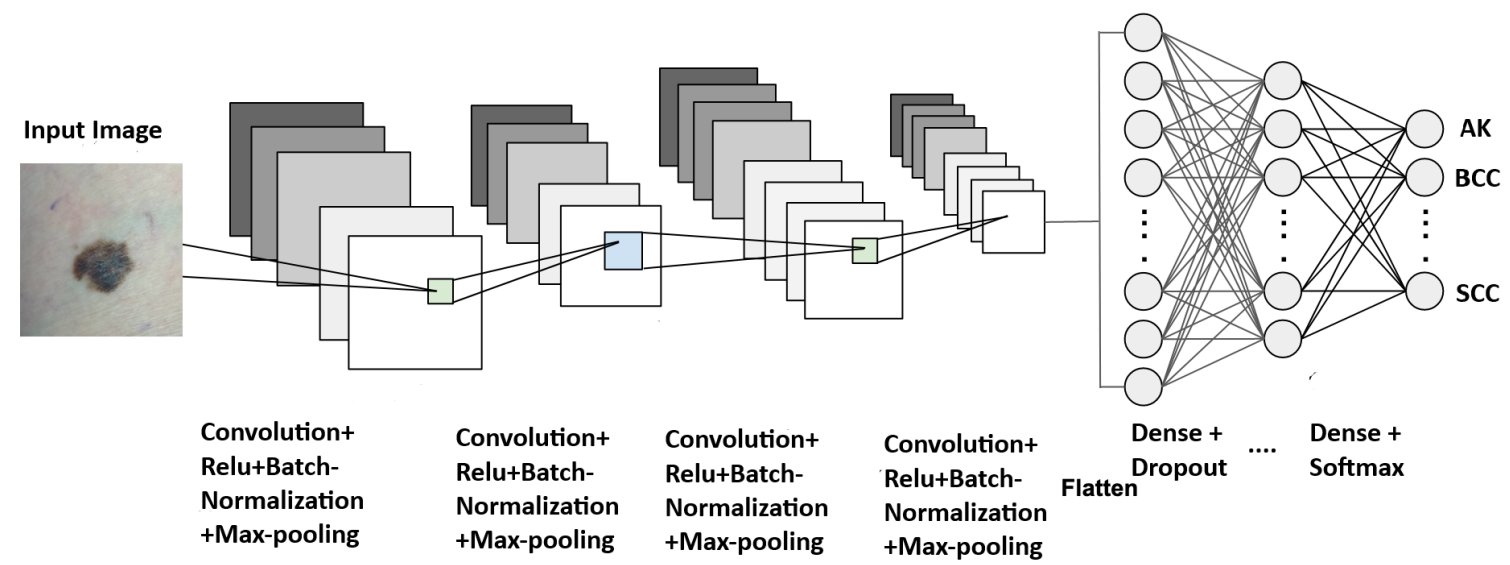

Figure 2: The architecture of propose deep CNN Model for Skin Cancer detection and classification.

image along to either $\mathrm{x}$-axis or $\mathrm{y}$-axis. Shearing means shifting one part of an image to any parallel side. Resizing an image to a given size is scaling. Table 1 contains a summary of the augmentation parameters. After applying all the transformations, each image normalized. After augmenting our dataset, we received in a total of 5600 images. Then we split it into training and testing sets. In our training phase, 4480 images $(80 \%$ of the dataset) used for model learning means training and validation. We kept 1120 images (20\% of the dataset) for testing and evaluating the model.

\subsection{Proposed Model}

Different types of deep learning architectures are commonly used for image and video classification, while recent methods focusing on deep CNN models for identifying and classifying 2D and 3D images [23] [24] [25] [26]. Due to memory limitations, it is computationally expensive to learn many trainable parameters and arithmetic operations [27]. It motives us to work on a deep learning-based method to detect and classify skin cancer. Our proposed model used an input layer corresponding to $224 \times 224$ input image with three input channels. In this architecture, we used the first convolutional layer from the input image with two and used ReLU activation function to activate every convolution layer. We used batch normalization, which normalizes the feature extraction and reduces data variance. It is used after ReLU (1) activation function and before max-pooling. It reduces the spatial dimensionality of the extracted feature maps. Then we used the output filter size as 16 with $7 \times 7$ kernel size followed by $2 \times 2$ size max-pooling layer with stride 2 . Then added three convolutional blocks, stacked over the first block, each having a $3 \times 3$ kernel size with $32,64,128$ and filters sequentially with ReLU activation function with corresponding batch-normalization and $2 \times 2$ max-pooling layers with stride 2 . Table 2 shows the summary of the proposed model with layers, configuration, parameters and output shape.

$$
\operatorname{RELU}(x)=\operatorname{MAX}(0, X)
$$

The outputs from these layers are flattened and connected with three fully connected layers: dense layers and dropout layers. We used 25\% of first two dropout layers and 50\% used for last dropout layer and the first two dense layers used in 256 and another dense layer used in 128, followed by Softmax (2) output layer activation function with probability images for each of the class.

$$
\operatorname{Softmax}\left(\left(x_{i}\right)\right)=\frac{\exp \left(x_{i}\right)}{\sum_{j} \exp \left(x_{j}\right)}
$$

It must be designed with the appropriate loss function and optimizer before being used for training. Adam was used as an optimizer because it has a lower computational cost, uses less memory, and is unaffected by gradient re-scaling. This addresses concerns like large data sets, hyper-parameters, noisy data, insufficient gradients, and non-stationary problems that involve finetuning. We used categorical cross-entropy as a loss function, which is defined as an $\mathrm{n}$-dimensional vector.

\subsection{Pre-train Models}

GoogleNet [28], and MobileNet [29] are one of the most popular pre-train approaches in Deep Learning and Transfer Learning that is being used by researchers broadly [31] [32]. We also used these two pre-train models that consist of 48 layers and 28 layers, respectively. These models are trained on large ImageNet dataset with 1000 classes and perform well. GoogleNet [28] has learned multi-level feature representation for a wide range of images. We use it with pre-train weight, and our images were $299 \times 299$. The last layer consists of an GlobalAverage, a Pooling2D layer and two Dense layers. SGD optimizer was applied and Binary Cross Entropy was used as the loss function [30]. Another image classification model is MobileNet [29]. We use 
Table 2: The summary of the proposed model with layers, configuration and output shape.

\begin{tabular}{|c|c|c|}
\hline Layers & Configuration & Output Shape \\
\hline Conv2D & $\begin{array}{l}224 \times 224 \times 16 ; \text { ker- } \\
\text { nel size: } 7 \times 7 \text {; stride: } \\
2 \text {; activation function: } \\
\text { RELU }\end{array}$ & $224 \times 224 \times 16$ \\
\hline BatchNormalization & - & $224 \times 224 \times 16$ \\
\hline Max-pooling2D & $\begin{array}{l}\text { Kernel size: } 2 \times 2 \text {; } \\
\text { stride: } 2 \text {; }\end{array}$ & $112 \times 112 \times 32$ \\
\hline Conv2D & $\begin{array}{l}112 \times 112 \times 32 \text {; ker- } \\
\text { nel size: } 3 \times 3 \text {; stride: } \\
2 \text {; activation function: } \\
\text { RELU }\end{array}$ & $112 \times 112 \times 32$ \\
\hline BatchNormalization & - & $112 \times 112 \times 32$ \\
\hline Max-pooling2D & $\begin{array}{l}\text { Kernel size: } 2 \times 2 \text {; } \\
\text { stride: } 2 \text {; }\end{array}$ & $56 \times 56 \times 64$ \\
\hline Conv2D & $\begin{array}{l}56 \times 56 \times 64 ; \text { kernel } \\
\text { size: } 3 \times 3 ; \text { stride: } \\
1 \text {; activation function: } \\
\text { RELU }\end{array}$ & $56 \times 56 \times 64$ \\
\hline BatchNormalization & - & $56 \times 56 \times 64$ \\
\hline Max-pooling2D & $\begin{array}{l}\text { Kernel size: } 2 \times 2 \text {; } \\
\text { stride: } 2 \text {; }\end{array}$ & $28 \times 28 \times 128$ \\
\hline Conv2D & $\begin{array}{l}28 \times 28 \times 128 \text {; kernel } \\
\text { size: } 3 \times 3 \text {; stride: } \\
2 \text {; activation function: } \\
\text { RELU }\end{array}$ & $28 \times 28 \times 128$ \\
\hline BatchNormalization & - & $28 \times 28 \times 128$ \\
\hline Max-pooling2D & $\begin{array}{l}\text { Kernel size: } 2 \times 2 \text {; } \\
\text { stride: } 2 \text {; }\end{array}$ & $14 \times 14 \times 256$ \\
\hline Flatten & - & 4096 \\
\hline Dense & - & 256 \\
\hline Dropout & 0.25 & 256 \\
\hline Dense & - & 128 \\
\hline Dropout & 0.25 & 128 \\
\hline Dense & - & 64 \\
\hline Dropout & 0.50 & 64 \\
\hline Dense & $\begin{array}{l}\text { activation: Softmax; } \\
\text { unit: } 4\end{array}$ & 4 \\
\hline
\end{tabular}

this with pre-train weight and $224 \times 224$ input images. Here, the last layer consists a Dense layer with softmax activation function. SGD optimizer was applied and Binary Cross Entropy was used as the loss function. After that, we use our augmented images to train and test those pre-trained models. We used a Dense layer with softmax activation function as the last layer. Adam optimizer was applied and Categorical Cross Entropy was used as the loss function. These pre-trained models decreased the requirement of computational power and saved time for training the model. Table 3 shows the two pre-train model's training details and our proposed model.

\subsection{Evaluation Metrics}

For measuring our model's performance, we evaluated it to observe which model gives the highest accuracy by predicting the sample data [33] [34]. We calculated accuracy, precision, recall, Specificity, Negative Predictive Rate and False Discovery Rate by using the True Positive (TP), True Negative (TN), False Positive (FP), False Negative (FN) values. We also observed the con-
Table 3: Training details of three deep learning models (two pre-train models and our proposed model).

\begin{tabular}{|c|c|c|c|}
\hline Training Details & GoogleNet & MobileNet & $\begin{array}{l}\text { Proposed } \\
\text { model }\end{array}$ \\
\hline $\begin{array}{l}\text { Data Augmenta- } \\
\text { tion }\end{array}$ & Yes & Yes & Yes \\
\hline $\begin{array}{l}\text { Transfer Learn- } \\
\text { ing }\end{array}$ & Yes & Yes & No \\
\hline Last layer & $\begin{array}{l}\text { GlobalAverage- } \\
\text { Pooling2D } \\
\text { Dense (1024, } \\
\text { activation = } \\
\text { relu) Dense } \\
(4, \text { activation } \\
=\text { sigmoid })\end{array}$ & $\begin{array}{l}\text { Dense }(4, \\
\text { activation }= \\
\text { softmax })\end{array}$ & $\begin{array}{l}\text { Dense } \\
(4, \text { acti- } \\
\text { vation = } \\
\text { softmax })\end{array}$ \\
\hline $\begin{array}{l}\text { Feature Extrac- } \\
\text { tion Enabled }\end{array}$ & Yes & No & Yes \\
\hline $\begin{array}{l}\text { Classification En- } \\
\text { abled }\end{array}$ & Yes & Yes & Yes \\
\hline Optimizer & SGD & SGD & ADAM \\
\hline Loss Function & $\begin{array}{l}\text { Binary Cross- } \\
\text { Entropy }\end{array}$ & $\begin{array}{l}\text { Binary } \\
\text { Cross- } \\
\text { Entropy }\end{array}$ & $\begin{array}{l}\text { Categorica } \\
\text { Cross- } \\
\text { Entropy }\end{array}$ \\
\hline $\begin{array}{l}\text { Number of } \mathrm{Pa}- \\
\text { rameters }\end{array}$ & $23,909,160$ & $2,259,265$ & $14,050,501$ \\
\hline $\begin{array}{l}\text { Number of Train- } \\
\text { able Parameters }\end{array}$ & $23,874,727$ & $2,225,153$ & $13,142,281$ \\
\hline
\end{tabular}

fusion matrix for predicting each class [35]. Following equations, 3 , 8 were used for evaluating the results.

$$
\begin{gathered}
\text { Accuracy }=\frac{(T P+T N)}{(T P+T N+F P+F N)} \\
\text { Recall/Sensitivity }=\frac{(T P)}{(F N+T P)} \\
\text { Precision }=\frac{(T P)}{(T P+F P)} \\
\text { NegativePredictiveValue }=\frac{(T N)}{(T N+F N)} \\
\text { FalseDiscoveryRate }=\frac{(F P)}{F P+T P} \\
\text { Specificity }=\frac{(T N)}{(F P+T N)}
\end{gathered}
$$

\section{RESULTS AND DISCUSSION}

\subsection{Experimental Setup}

To train our proposed model, we used Google Colab GPU platform using python environment. For training, We compiled our proposed model with ADAM optimizer with a learning rate of 0.001 , and the batch size was 32. After that, we train our model for 50 epochs with 'categorical cross-entropy' as the loss function. We measured the model train and validation graph by observing the loss function. We used pre-trained models that were fine-tuned to adjust some parameters for training. 

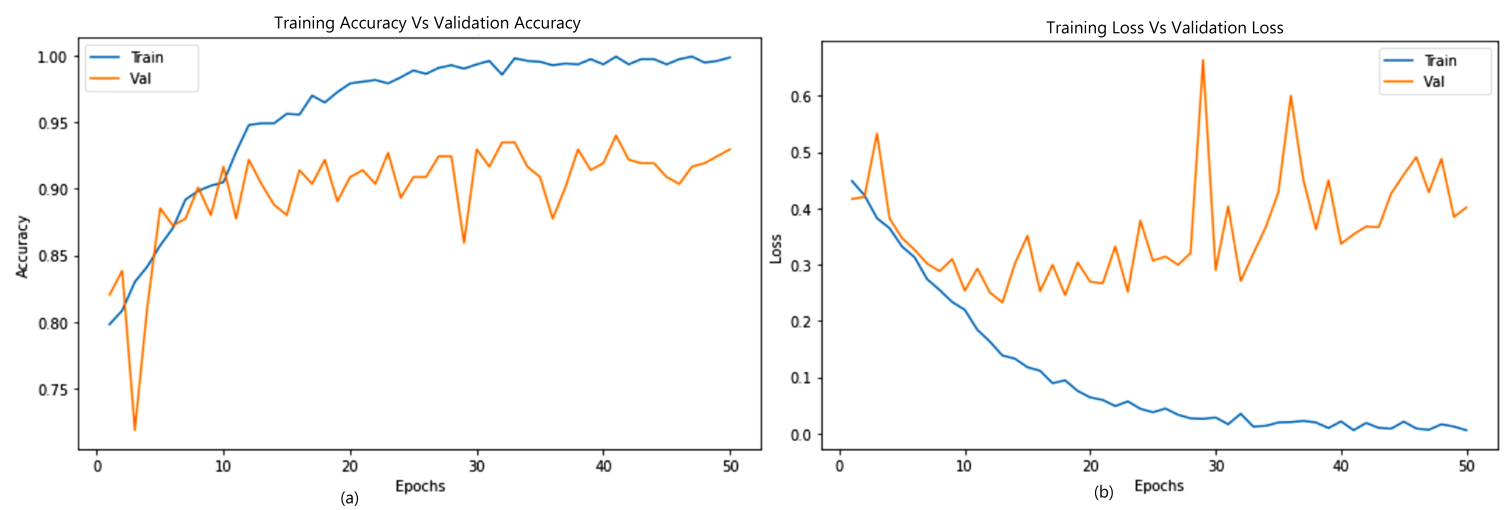

Figure 3: (a) Accuracy, and (b) Loss Graph of our proposed model.

\subsection{Results}

Table 4 represents the confusion matrix of our proposed model. It shows a tabular way of the performance of our model. Each entry in a confusion matrix denotes the number of predictions made by the model where it classified the classes correctly or incorrectly. Here the rows and columns indicate actual labels and predicted labels respectively. Depending on the number of classes, The size of our confusion matrix became $4 \times 4$. Table 5 shows the performance evaluation that

Table 4: The Confusion Matrix (CM) of our proposed model.

\begin{tabular}{|c|c|c|c|c|}
\hline Classes & AK & BCC & MM & SCC \\
\hline AK & $\mathbf{2 5 8}$ & 5 & 9 & 8 \\
\hline BCC & 3 & $\mathbf{2 6 9}$ & 3 & 5 \\
\hline MM & 15 & 2 & $\mathbf{2 5 6}$ & 7 \\
\hline SCC & 9 & 13 & 11 & $\mathbf{2 4 7}$ \\
\hline
\end{tabular}

includes Precision, Recall, Accuracy, Negative Predictive Value and False Discovery Rate for four different skin cancer classes of our proposed model. Equation 3 to 7 was used for calculating these values. The different evaluation metrics indicate the overall performance of our model. We have got the highest accuracy $97.23 \%$ in Basal Cell Carcinoma (BCC). Basal Cell Carcinoma acquired the highest values in other metrics such as Precision, Recall and Negative Predictive Value.

Table 5: Performance Evaluation of our proposed Model.

\begin{tabular}{|c|c|c|c|c|c|}
\hline Types & Precision & Recall & Accuracy & NPV & F-Rate \\
\hline AK & 0.92 & 0.90 & $95.63 \%$ & 0.97 & 0.08 \\
\hline BCC & 0.99 & 0.93 & $97.23 \%$ & 0.98 & 0.04 \\
\hline MM & 0.91 & 0.91 & $95.80 \%$ & 0.97 & 0.09 \\
\hline SCC & 0.88 & 0.92 & $95.27 \%$ & 0.98 & 0.12 \\
\hline
\end{tabular}

Figure 3 (a) describes the training and validation accuracy of our model. Also the training and validation loss is shown graphically in Fig. 3(b). Here, the blue line represents the training performance, and the orange line represents the validation performance. After training, we noticed there was a slight overfitting pattern. This happened due to the new dataset. The different classes had very similar characteristics which led the training to occur in an overfitting pattern.

Table 6: Different training options with performance of proposed model.

\begin{tabular}{|c|c|c|c|c|}
\hline Optimizers & Loss Function & Pre & Rec & Acc \\
\hline SGD & MSE & $85.42 \%$ & $81.65 \%$ & $89.97 \%$ \\
\hline SGD & cross entrophy & $85.74 \%$ & $85.36 \%$ & $90.68 \%$ \\
\hline ADAM & MSE & $88.55 \%$ & $87.81 \%$ & $92.32 \%$ \\
\hline ADAM & cross entrophy & $\mathbf{9 1 . 9 6 \%}$ & $\mathbf{9 1 . 9 7 \%}$ & $\mathbf{9 5 . 9 8 \%}$ \\
\hline
\end{tabular}

To get the best among all, we used SGD and ADAM optimizers with MSE and cross entropy loss functions. When we chose ADAM as the optimizer with cross entropy loss function, we got $95.98 \%$ accuracy with $91.96 \%$ precision and $91.97 \%$ recall. Table 6 depicts the performance of proposed model with different optimizers and loss functions.

\subsection{K-Fold Cross Validation}

$\mathrm{K}$-fold cross validation is used to know how accurately the model will predict if a new test set is used. Here $\mathrm{k}$ is the number of folds where each fold will be used as a test set. In this case, to evaluate the proposed model on our dataset, we used 5-fold cross validation. The first fold was used as the test set in the first iteration. Sequentially the next folds are used as test set in next four iterations. As we have 5600 images, each fold consists of 1120 images.

The confusion matrics for each fold is shown in figure 4. The confusion matrics for each fold is shown in figure 4. Here in each confusion matrix, each row is for actual values and column is for the predicted values of the model. True positive values refers to the prediction of the existence of skin cancer when it is actually 


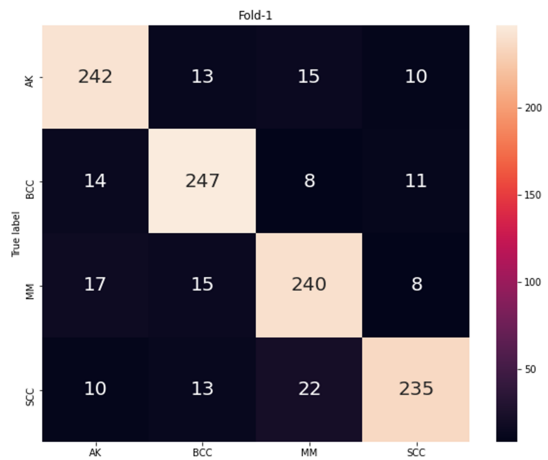

(a)

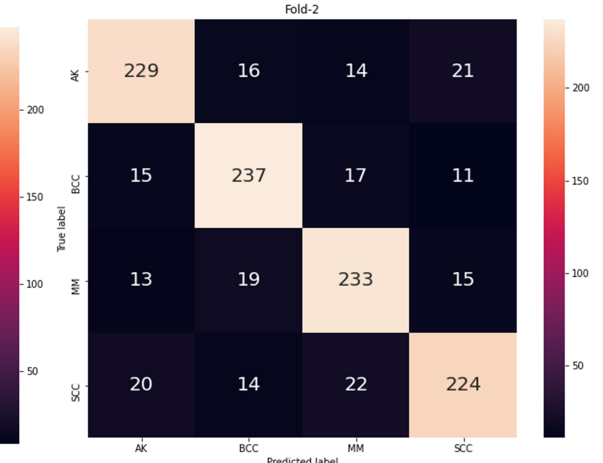

(b)

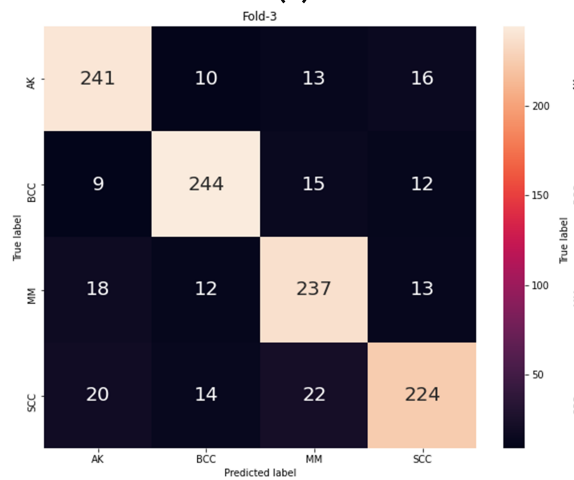

(c)

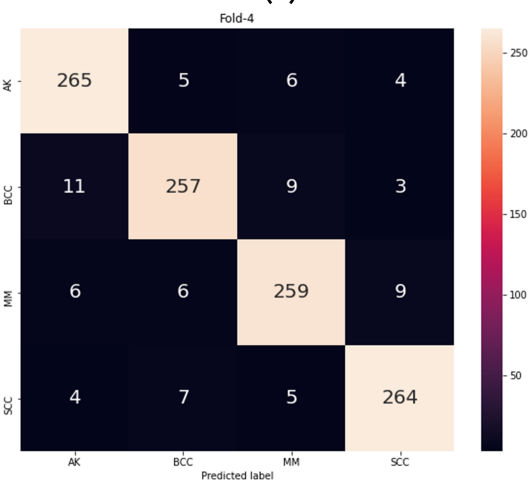

(d)

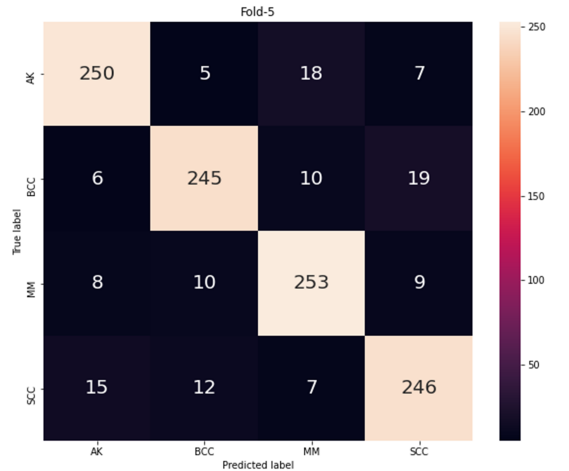

(e)

Figure 4: Confusion matrix of 5-fold cross validation process for each fold: (a) fold 1, (b) fold 2, (c) fold 3, (d) fold 4 , and (e) fold 5 .

present. In the first fold, the true positive values are 242, 247, 240, 235 for Actinic Keratosis, Basal Cell Carcinoma, Malignant Melanoma and Squamous Cell Carcinoma respectively. Similarly, the true positive values for next four folds are $(229,237,233,224),(241$, 244, 237, 224), (265, 257, 259, 264), (250, 245, 253, 246), respectively. So for each iteration, we can see the model prediction is quite well.

We also measured precision and recall for each fold. Folds were shuffled 5 times and the measures are given in table 7. After observing these values for each fold, we got an average of $87.08 \%$ and $87.16 \%$ precision and recall values respectively. The average accuracy of 5 folds is $93.50 \%$. From this measurements, we can say that overfitting is present.
Table 7: Measurement of 5-fold cross validation of this dataset.

\begin{tabular}{|c|c|c|c|}
\hline Fold & Precision (\%) & Recall (\%) & Accuracy (\%) \\
\hline Fold-1 & 86.03 & 86.25 & 93.04 \\
\hline Fold-2 & 82.53 & 82.47 & 91.21 \\
\hline Fold-3 & 84.59 & 84.75 & 92.23 \\
\hline Fold-4 & 93.48 & 93.25 & 96.65 \\
\hline Fold-5 & 88.76 & 89.09 & 94.37 \\
\hline Average & $\mathbf{8 7 . 0 8}$ & $\mathbf{8 7 . 1 6}$ & $\mathbf{9 3 . 5 0}$ \\
\hline
\end{tabular}

\subsection{Comparison}

The performance comparison among the Deep Learning models was made using Sensitivity, Specificity, Pre- 
Table 8: Comparison between Deep Learning Models

\begin{tabular}{|c|c|c|c|c|c|}
\hline Dataset type & Models & Sensitivity & Specificity & Precision & Accuracy \\
\hline \multirow{3}{*}{ Without Augmentation } & GoogleNet [28] & $79.81 \%$ & $84.23 \%$ & $79.57 \%$ & $84.66 \%$ \\
& MobileNet [29] & $81.09 \%$ & $84.97 \%$ & $81.55 \%$ & $85.48 \%$ \\
& Proposed Model & $\mathbf{8 3 . 1 8 \%}$ & $\mathbf{8 8 . 9 3 \%}$ & $\mathbf{8 4 . 7 2 \%}$ & $\mathbf{8 7 . 3 6 \%}$ \\
\hline \multirow{3}{*}{ With Augmentation } & GoogleNet [28] & $90.75 \%$ & $94.91 \%$ & $88.38 \%$ & $94.21 \%$ \\
& MobileNet [29] & $90.23 \%$ & $95.66 \%$ & $90.73 \%$ & $94.85 \%$ \\
& Proposed Model & $\mathbf{9 1 . 9 7 \%}$ & $\mathbf{9 7 . 3 3 \%}$ & $\mathbf{9 1 . 9 6 \%}$ & $\mathbf{9 5 . 9 8 \%}$ \\
\hline
\end{tabular}

Table 9: Comparison our result with the state of the arts.

\begin{tabular}{|c|c|c|c|c|}
\hline Approaches & Methods & Dataset Size & Classes & Accuracy \\
\hline Moussa et al. [39] & k-NN & 15 & 2 & $89 \%$ \\
\hline Dubal et al. [36] & NN & 463 & 6 & $76.90 \%$ \\
\hline Alquran et al. [37] & SVM & 11 & 1 & $92.1 \%$ \\
\hline Victor et al. [38] & KNN & 1000 & 2 & $93.70 \%$ \\
\hline Milton et al. [40] & DNN & 2000 & 7 & $76 \%$ \\
\hline Ulzii et al. [22] & CNN & 3753 & 4 & $94.2 \%$ \\
\hline Proposed model & Deep CNN & $\mathbf{5 6 0 0}$ & $\mathbf{4}$ & $\mathbf{9 5 . 9 8 \%}$ \\
\hline
\end{tabular}

cision and Accuracy. It shows that our proposed model performs better than pre-train models, GoogleNet and MobileNet. Table 8 demonstrates the results. At first, the pre-trained models and our proposed models are trained with images that are not augmented. Here we can see that our model performed better than other two models with an accuracy of $87.36 \%$. To improve the accuracy, in the next approach, we trained the model with augmented images. This time the accuracy of our proposed model is $95.98 \%$.

Table 9 shows the comparison of our model with previous works. After applying augmentation, our used dataset was the most enriched of all the previous works done before. Also, our proposed model outperformed all the previous researches in classifying different types of Skin Cancer. Various Neural Networks, SVM, KNN, and Convolutional Neural Networks have been used among the earlier works. The size and the feature of the dataset also had varieties. Milton et al. [40] had worked with the most established dataset with 2000 images in 2 classes. The work focused on multiple pre-train networks and was able to secure at best $76 \%$ accuracy. On the other hand, Victor et al. [38] used 1000 images with binary classes and used classical machine learning algorithm KNN and SVM. They were able to secure 93.70\% on their work. Our work surpassed previous results by both the dataset and accuracy.

\section{CONCLUSIONS}

In this paper, we have collected a dataset of four types of skin cancer and used augmentation techniques to increase our dataset. We proposed a deep learning-based method to classify skin cancer. We used some regularization methods like batch normalization to avoid overfitting and used convolution, max pooling, dropout, and fully connected layers. Our proposed model achieved $96.98 \%$ accuracy for four types of skin cancer that is the state of arts. This accuracy is higher than two other pre-train models, GoogleNet and MobileNet. Five-fold cross-validation is used to verify the proposed model with this dataset. In summation, our proposed model provided better comparable performance to the existing state-of-the-art methods in terms of accuracy, precision, recall, sensitivity, and specificity. It is also simple and lightweight architecture than other models. In future, a light architecture can be designed without compromising the accuracy to detect skin cancer by minimizing computational complexity.

\section{REFERENCES}

[1] Craythorne, E. and Al-Niami, F., 2017. Skin cancer. Medicine, 45(7), pp.431-434.

[2] https://www.skincancer.org/skin-cancerinformation/squamous-cell-carcinoma/ [Last visited: 12-01-2021]

[3] Siegel, J.A., Korgavkar, K. and Weinstock, M.A., 2017. Current perspective on actinic keratosis: a review. British Journal of Dermatology, 177(2), pp.350-358.

[4] Garbe, C., Peris, K., Hauschild, A., Saiag, P., Middleton, M., Bastholt, L., Grob, J.J., Malvehy, J., Newton-Bishop, J., Stratigos, A.J. and Pehamberger, H., 2016. Diagnosis and treatment of melanoma. European Journal of Cancer, 63, pp.201-217. 
[5] https://cancerstatisticscenter.cancer.org [last visited: 12-01-2021]

[6] Vocaturo, E., Perna, D. and Zumpano, E., 2019, November. Machine Learning Techniques for Automated Melanoma Detection. In 2019 IEEE International Conference on Bioinformatics and Biomedicine (BIBM) (pp. 2310-2317). IEEE.

[7] Ansari, U.B. and Sarode, T., 2017. Skin cancer detection using image processing. Int Res J Eng Technol, 4(4), pp.2875-2881.

[8] Stefanczyk, M. and Bochenski, T., 2020. Mixing deep learning with classical vision for object recognition. Journal of WSCG, Vol.28, No.1-2, 2020.

[9] Khan, M.Q., Hussain, A., Rehman, S.U., Khan, U., Maqsood, M., Mehmood, K. and Khan, M.A., 2019. Classification of Melanoma and Nevus in Digital Images for Diagnosis of Skin Cancer. IEEE Access, 7, pp.90132-90144.

[10] Gour, N. and Khanna, P., 2021. Multi-class multilabel ophthalmological disease detection using transfer learning based convolutional neural network. Biomedical Signal Processing and Control, 66, p. 102329

[11] Esteva, A., Kuprel, B., Novoa, R.A., Ko, J., Swetter, S.M., Blau, H.M. and Thrun, S., 2017. Dermatologist-level classification of skin cancer with deep neural networks. nature, 542(7639), pp.115-118.

[12] Junayed, M.S., Sakib, A.N.M., Anjum, N., Islam, M.B. and Jeny, A.A., 2020, December. EczemaNet: A Deep CNN-based Eczema Diseases Classification. In 2020 IEEE 4th International Conference on Image Processing, Applications and Systems (IPAS) (pp. 174-179). IEEE.

[13] Wu, Z., Zhao, S., Peng, Y., He, X., Zhao, X., Huang, K., Wu, X., Fan, W., Li, F., Chen, M. and Li, J., 2019. Studies on different CNN algorithms for face skin disease classification based on clinical images. IEEE Access, 7, pp.66505-66511.

[14] Garg, N., Sharma, V. and Kaur, P., 2018. Melanoma skin cancer detection using image processing. In Sensors and Image Processing (pp. 111-119). Springer, Singapore.

[15] Sreelatha, T., Subramanyam, M.V. and Prasad, M.G., 2019. Early detection of skin cancer using melanoma segmentation technique. Journal of medical systems, 43(7), p.190.

[16] Pham, T.C., Luong, C.M., Visani, M. and Hoang, V.D., 2018, March. Deep CNN and data augmentation for skin lesion classification. In Asian Conference on Intelligent Information and Database Systems (pp. 573-582). Springer, Cham.
[17] Yap, J., Yolland, W. and Tschandl, P., 2018. Multimodal skin lesion classification using deep learning. Experimental dermatology, 27(11), pp.12611267.

[18] Jianu, S.R.S., Ichim, L. and Popescu, D., 2019, March. Automatic diagnosis of skin cancer using neural networks. In 2019 11th International Symposium on Advanced Topics in Electrical Engineering (ATEE) (pp. 1-4). IEEE.

[19] Hosny, K.M., Kassem, M.A. and Foaud, M.M., 2018, December. Skin cancer classification using deep learning and transfer learning. In 2018 9th Cairo International Biomedical Engineering Conference (CIBEC) (pp. 90-93). IEEE.

[20] Mohamed, E.H. and El-Behaidy, W.H., 2019, December. Enhanced Skin Lesions Classification Using Deep Convolutional Networks. In 2019 Ninth International Conference on Intelligent Computing and Information Systems (ICICIS) (pp. 180188). IEEE.

[21] Nahata, H. and Singh, S.P., 2020. Deep learning solutions for skin cancer detection and diagnosis. In Machine Learning with Health Care Perspective (pp. 159-182). Springer, Cham.

[22] Dorj, U.O., Lee, K.K., Choi, J.Y. and Lee, M., 2018. The skin cancer classification using deep convolutional neural network. Multimedia Tools and Applications, 77(8), pp.9909-9924.

[23] Waheed, A., Goyal, M., Gupta, D., Khanna, A., Hassanien, A.E. and Pandey, H.M., 2020. An optimized dense convolutional neural network model for disease recognition and classification in corn leaf. Computers and Electronics in Agriculture, 175 , p. 105456.

[24] Junayed, M.S., Jeny, A.A., Atik, S.T., Neehal, N., Karim, A., Azam, S. and Shanmugam, B., 2019, July. AcneNet-A deep CNN based classification approach for acne classes. In 2019 12th International Conference on Information \& Communication Technology and System (ICTS) (pp. 203-208). IEEE.

[25] Anwar, S.M., Majid, M., Qayyum, A., Awais, M., Alnowami, M. and Khan, M.K., 2018. Medical image analysis using convolutional neural networks: a review. Journal of medical systems, 42(11), pp.1-13.

[26] Yu, H., Yang, L.T., Zhang, Q., Armstrong, D. and Deen, M.J., 2021. Convolutional neural networks for medical image analysis: state-of-theart, comparisons, improvement and perspectives. Neurocomputing.

[27] Zhang, Q., Bai, C., Liu, Z., Yang, L.T., Yu, H., Zhao, J. and Yuan, H., 2020. A GPU-based residual network for medical image classification 
in smart medicine. Information Sciences, 536, pp.91-100.

[28] Szegedy, C., Vanhoucke, V., Ioffe, S., Shlens, J. and Wojna, Z., 2016. Rethinking the inception architecture for computer vision. In Proceedings of the IEEE conference on computer vision and pattern recognition (pp. 2818-2826).

[29] Howard, A.G., Zhu, M., Chen, B., Kalenichenko, D., Wang, W., Weyand, T., Andreetto, M. and Adam, H., 2017. Mobilenets: Efficient convolutional neural networks for mobile vision applications. arXiv preprint arXiv:1704.04861.

[30] Kumar, A., Sarkar, S. and Pradhan, C., 2020. Malaria Disease Detection Using CNN Technique with SGD, RMSprop and ADAM Optimizers. In Deep Learning Techniques for Biomedical and Health Informatics (pp. 211-230). Springer, Cham.

[31] Gour, N. and Khanna, P., 2021. Multi-class multilabel ophthalmological disease detection using transfer learning based convolutional neural network. Biomedical Signal Processing and Control, 66, p.102329.

[32] Zwettler, G.A., Holmes III, D.R. and Backfrieder, W., 2020. Strategies for Training Deep Learning Models in Medical Domains with Small Reference Datasets. Journal of WSCG. 2020, vol. 28, no. 1-2, p. 37-46.

[33] Junayed, M.S., Jeny, A.A., Neehal, N., Ahmed, E. and Hossain, S.A., 2018, December. Incept-N: A Convolutional Neural Network Based Classification Approach for Predicting Nationality from Facial Features. In International Conference on Recent Trends in Image Processing and Pattern Recognition (pp. 466-475). Springer, Singapore.

[34] Macatangay, J.M.A., Ruiz Jr, C.R. and Usatine, R.P., 2017. A primary morphological classifier for skin lesion images. 25th International Conference in Central Europe on Computer Graphics, Visualization and Computer Visionin co-operation with EUROGRAPHICS Association, p. 55-64.

[35] Jeny, A.A., Junayed, M.S., Ahmed, I., Habib, M.T. and Rahman, M.R., 2019, December. FoNetLocal Food Recognition Using Deep Residual Neural Networks. In 2019 International Conference on Information Technology (ICIT) (pp. 184189). IEEE.

[36] Dubal, P., Bhatt, S., Joglekar, C. and Patil, S., 2017, November. Skin cancer detection and classification. In 2017 6th international conference on electrical engineering and informatics (ICEEI) (pp. 1-6). IEEE.

[37] Alquran, H., Qasmieh, I.A., Alqudah, A.M., Alhammouri, S., Alawneh, E., Abughazaleh, A. and
Hasayen, F., 2017, October. The melanoma skin cancer detection and classification using support vector machine. In 2017 IEEE Jordan Conference on Applied Electrical Engineering and Computing Technologies (AEECT) (pp. 1-5). IEEE.

[38] Victor, A. and Ghalib, M., 2017. Automatic detection and classification of skin cancer. International Journal of Intelligent Engineering and Systems, 10(3), pp.444-451.

[39] Moussa, R., Gerges, F., Salem, C., Akiki, R., Falou, O. and Azar, D., 2016, October. Computeraided detection of Melanoma using geometric features. In 2016 3rd Middle East Conference on Biomedical Engineering (MECBME) (pp. 125128). IEEE.

[40] Milton, M.A.A., 2019. Automated skin lesion classification using ensemble of deep neural networks in isic 2018: Skin lesion analysis towards melanoma detection challenge. arXiv preprint arXiv:1901.10802. 